\title{
Gastroesophageal Cancer: Prognostic Factors and Treatment Results
}

Prochorov $\mathrm{AV}^{*}$, Labunec $\mathrm{IN}^{1}$, Shepetjko $\mathrm{MN}^{1}$, Mavrichev $\mathrm{YV}^{2}$ and Gedrevich $\mathrm{ZE}^{1}$

${ }^{1}$ Department of Oncology of Belarusian State Medical University, Minsk, Belarus

${ }^{2}$ Public Health Institution, Minsk City Clinical Oncology Hospital, Minsk, Belarus

${ }^{*}$ Corresponding author: Prochorov AV, Head of Department of Oncology, Belarusian State Medical University, Dzerzhinski ave., 83, Minsk, Belarus, Fax and Tel: +375172902971, E-mail: aprokharau@gmail. com

Citation: Prochorov AV, Labunec IN, Shepetjko MN, Mavrichev YV, Gedrevich ZE (2016) Gastroesophageal Cancer: Prognostic Factors and Treatment Results. J Cancer Sci Clin Oncol 3(1): 104. doi: 10.15744/23946520.3.104

Received Date: April 18, 2016 Accepted Date: June 07, 2016 Published Date: June 09, 2016

\section{Abstract}

The study presents the remote results of surgical treatment of 329 patients with cancer of gastroesophageal localization. Three 3-year survival rate is $37.1 \%$, the 5 -year survival rate is $26.2 \%$. Prognosis after the surgery depends primarily on the extent of the tumour spread. In all types of surgeries the survival rate of patients without lymph nodes metastasis is twice as high as that of patients with metastatic spread to lymph nodes. The amount of the affected lymph nodes does not affect overall as well as relapse-free survival. Combined and palliative resections may procedure the 5-year survival rate at the level of only $10-15 \%$.

Keywords: Gastroesophageal cancer; Surgical treatment; Survival rate; Remote treatment results

\section{Introduction}

Despite the downward trend in the gastric cancer incidence rate worldwide, every year in Europe (ESMO, 2012) approximately 140000 new cases are still being diagnosed, resulting in up to 107000 deaths from this pathology [1]. While the overall gastric cancer incidence rate is declining, there is a relative increase in the gastrooesophageal junction (GEJ) and gastric cardia cancer incidence rates [2,3]. A similar upward trend in the gastroesophageal cancer is recorded in Republic of Belarus. In 2014, according to the National Morbidity Survey of Belarus, gastric cancer was the third most common cancer, and the second leading cause of death from oncological pathology [4].

Surgery remains the main treatment modality for gastroesophageal cancer. Tumor of this localization is characterized by high grade malignancy and extremely strong potential for the fast metastasis into the abdomen and mediastinum lymph nodes [5-8]. As such, the complexity of the gastroesophageal cancer surgery is caused by the necessity to execute lymphadenectomy in two anatomical areas: mediastinum and abdomen $[6,9]$.

Despite the progress made in the significant reduction of postoperative complications and following mortality, the long- term results of the treatment cannot be considered satisfactory. Certainly, many surgeons have improved long-term outcomes of surgical treatment by performing the extended D2 and D3 lymph node dissections, thus achieving the 3-year survival rate at 44-47\% [1012]. However, the extremely low survival rates are still being observed in the groups of patients with infiltrating undifferentiated types of cancer and regional lymph nodes metastasis that developed by the time of the operation $[2,6]$. Definitely, the adequate dissection has a significant influence on the long-term outcome; however, it remains evident that an early diagnosis is the main factor in the achieving more satisfactory results of the surgical treatment. $[6,7,13]$. The purpose of this study was to examine the remote results of surgical treatment of gastroesophageal cancer depending on the most important prognostic factors such as the stage of disease with the extent and the depth of the tumor invasion, the lymph nodes metastasis, the type and the scope of surgery, the morphological type and the degree of differentiation of the tumor.

\section{Patients and Methods}

The study is based on the results of surgical treatment of 329 gastroesophageal cancer patients hospitalized by the Public Health Institution "Minsk City Clinical Oncology Hospital" in the period from 2002 to 2015. This group consisted of patients with tumors, which anatomical epicenters were located in the lower thoracic esophagus, or GEJ, or within the proximal $5 \mathrm{~cm}$ of stomach (i.e., cardia) with the tumor mass extending into GEJ or distal esophagus. All patients have undergone the transpleural gastric and esophagus resection by combined thoracolaparotomy access, as follows: in 155 cases - gastrectomy with resection of the lower third 
of the esophagus by Garlock-Osawa; in 96 cases - proximal gastrectomy with the lower third of the esophagus by Garlock-Osawa; in 78 cases - proximal gastrectomy with subtotal resection of the esophagus by Lewis. D2 dissections with excision of at least 16 lymph nodes were performed in all cases. In the cases of dissection by Lewis the procedure was complemented by removal of the paraesophageal and bifurcation lymph nodes. The average age of the patients under the study was 61.5 years (28 - 83 year old range) with men to women ratio - 2.6:1 (237 males, 92 females).

197 patients with stage pT2-4N1-3M0-1, including those treated with the R0-R2 resection, received the first- line adjuvant chemotherapy with the XELOX, CF, ECF regimen. In case of disease progression or recurrence, the second- and the third- line of chemotherapy were applied according to the recommendations of the multidisciplinary tumor board.

The survival of the patients was measured by the 3- and 5-year survival rates (adjusted for postoperative mortality) according to Kaplan-Meier method. A comparison of survival rates depending on various factors in groups was performed with the log-rank test. Multivariate statistical analysis of variables influencing the survival rate was conducted by the Cox proportional hazards model. For every factor the hazard ratio (HR) was calculated with $95 \%$ confidence interval (CI).

\section{Results}

Preoperatively and intraoperatively, $60 \%$ of the patients (197 cases) were diagnosed with single or multiple lymph node metastases, $15 \%$ of the patients (51 case) - with tumor invasion into adjacent anatomical structures - diaphragm, pancreas, liver, mesocolon (Table 1).

\begin{tabular}{|c|c|c|c|}
\hline Index T: abs. (\%) & Index N: abs. (\%) & Index M: abs. (\%) & Stage: abs. (\%) \\
\hline $\mathrm{T}_{1}-35(11 \%)$ & $\mathrm{N}_{0}-132(40 \%)$ & \multirow{2}{*}{$\mathrm{M}_{0}-287(87 \%)$} & $\mathrm{I}-71(22 \%)$ \\
\cline { 1 - 2 } $\mathrm{T}_{2}-114(35 \%)$ & $\mathrm{N}_{1}-102(31 \%)$ & & $\mathrm{II}-83(25 \%)$ \\
\hline $\mathrm{T}_{3}-129(39 \%)$ & $\mathrm{N}_{2}-65(20 \%)$ & \multirow{2}{*}{$\mathrm{M}_{1}-42(13 \%)$} & $\mathrm{III}-111(34 \%)$ \\
\cline { 1 - 2 } $\mathrm{T}_{4}-51(15 \%)$ & $\mathrm{N}_{3}-30(9 \%)$ & & $\mathrm{IV}-64(19 \%)$ \\
\hline
\end{tabular}

Table 1: Tumor extention and stage

Standard D2 lymph node dissection was performed in 246 cases (75\%); combined operations with resection of affected adjacent structures were performed in 83 cases (25\%). Based on the results of the histological examination of surgical biopsy material, in 298 cases (91\%) the surgeries were classified as radical (R0), and in 31 case (9.0\%) - as palliative (R1, R2). By histological structure, in 226 cases out of $329(69 \%)$ the gastroesophageal tumor was classified as adenocarcinoma in various degrees of differentiation (G1, G2, G3). Squamous cell carcinoma was diagnosed in 22\% (72 cases), and undifferentiated carcinoma - in 9\% (31 case).

Postoperative mortality in groups of operated patients reached 5.2\% (17 out of 329 cases): after gastrectomy with resection of the lower third of the esophagus by Garlock-Osawa - 3.9\% (6 cases out of 155); after proximal gastrectomy with the lower third of the esophagus by Garlock - Osawa - 5.2\% (5 cases out of 96); after proximal gastrectomy with subtotal resection of the esophagus by Lewis $-7.7 \%$ (6 cases out of 78$)$.

The combined 3-and 5-year survival rates, calculated on the entire group of operated patients, were $37.1 \%$ and $26.2 \%$, respectively. Admittedly, survival of gastroesophageal cancer patients after radical surgery influenced by a number of factors $[2,7,8]$. Some major prognostic variables are the stage of the disease with the depth of tumor invasion, presence or absence of metastatic spread into the lymph nodes, the scope and adequacy of surgical intervention. We analyzed the influence of the before mentioned factors on the life expectancy of the patients that have undergone the radical surgery.

The analysis revealed that the most powerful prognostic indicator for patients' survival after radical gastroesophageal cancer surgery is the presence or absence of the lymph nodes metastases. Out of all patients without the lymph nodes metastases, the 3and 5 -year observation period survived $59.2 \%$ and $43.6 \%$, respectively; in the group with the lymph nodes metastases, the 3 -and 5 -year observation period survived $26.2 \%$ and $18.4 \%$, respectively. Moreover, the survival rate in the latter did not depend on the number of affected lymph nodes N1- N3 (Figure 1).

Out of 298 cases of gastric and esophageal resection classified as radical (R0), 3-and 5- year observation period survived 39.3\% and $29.1 \%$ patients, respectively. Out of all the cases classified as palliative surgery, 3-and 5-year survival rate were 9.4\% (Figure 2).

Less profound but still statistically significant long-term results were received after analyzing the outcomes of combined operations with resection of tumor affected adjacent organs as compared to those of the standard surgical procedures: the 3-year survival rates were $21.1 \%$ and $43.7 \%$, respectively; the 5 -year survival rates $-15.2 \%$ and $31.3 \%$, respectively (Figure 3 ).

Survival analysis by the type and extent of surgery revealed that higher 3-and 5-year survival rates were in the group of patients who undergone proximal resection $-38.6 \%$ and $32.5 \%$, respectively (Figure 4 ). 


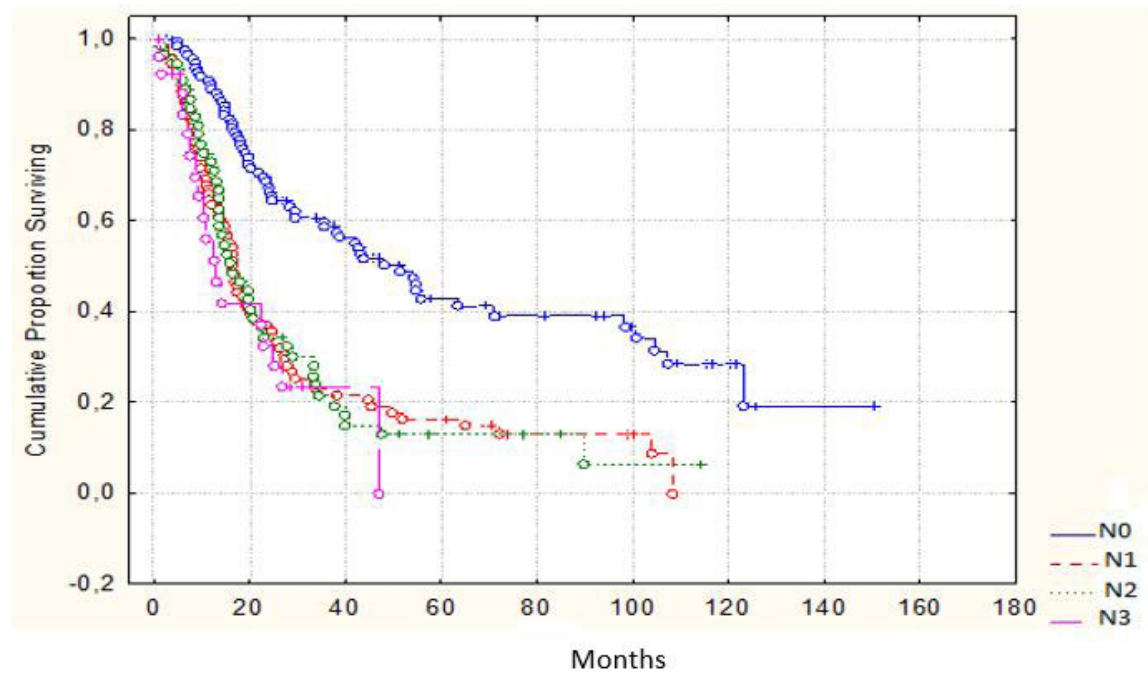

Figure 1: Survival according to the lymphatic metastasis $(\mathrm{P}<0.05)$

Cumulative Hroportion Surviving (Kaplan-Meier)

- Complete + Censored

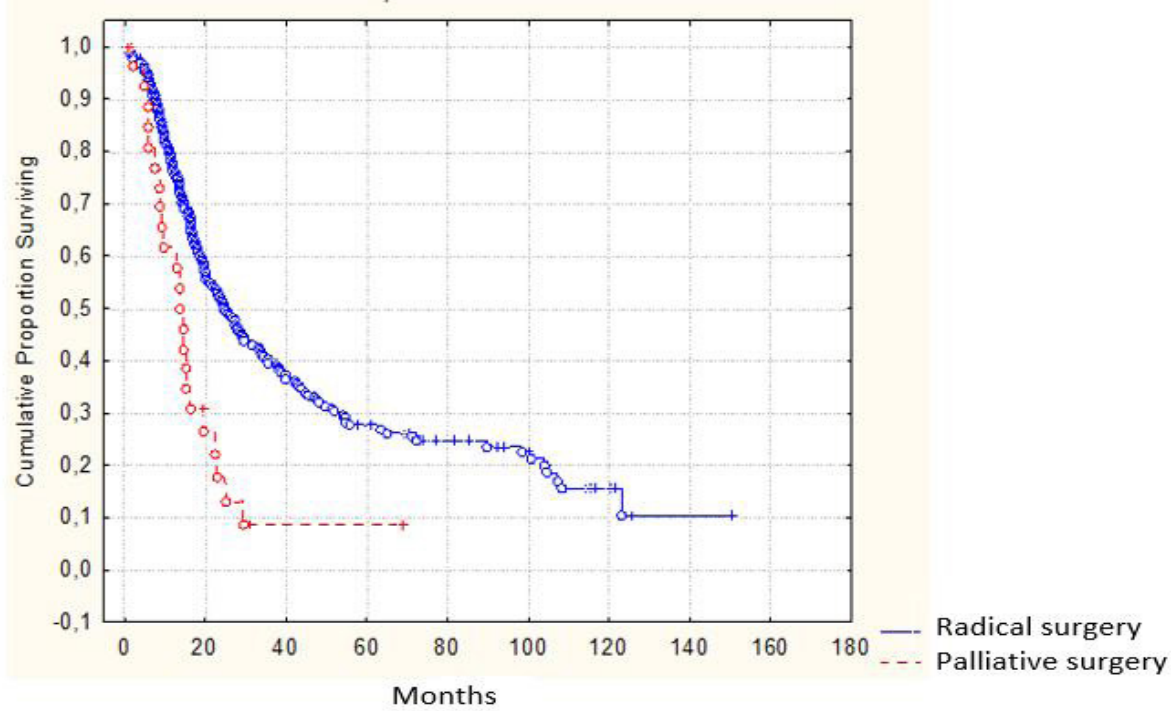

Figure 2: Survival according to the operation type $(\mathrm{P}<0.05)$

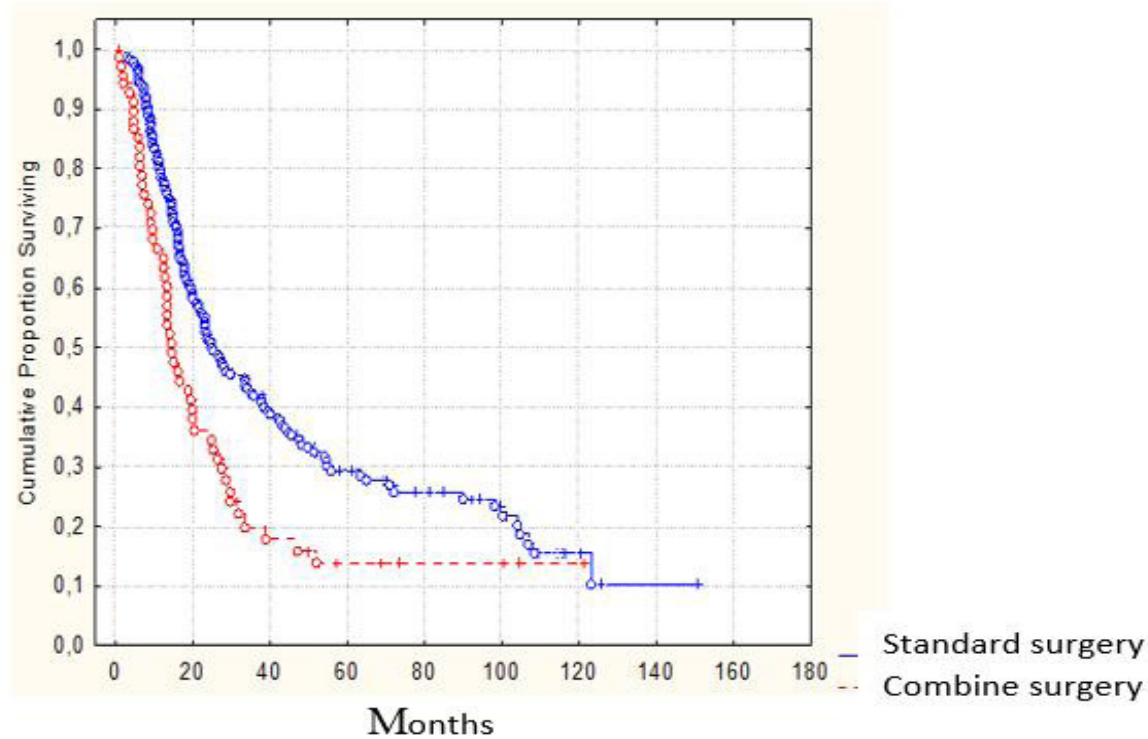

Figure 3: Survival according to the scope of the surgery $(\mathrm{P}>0.05)$ 


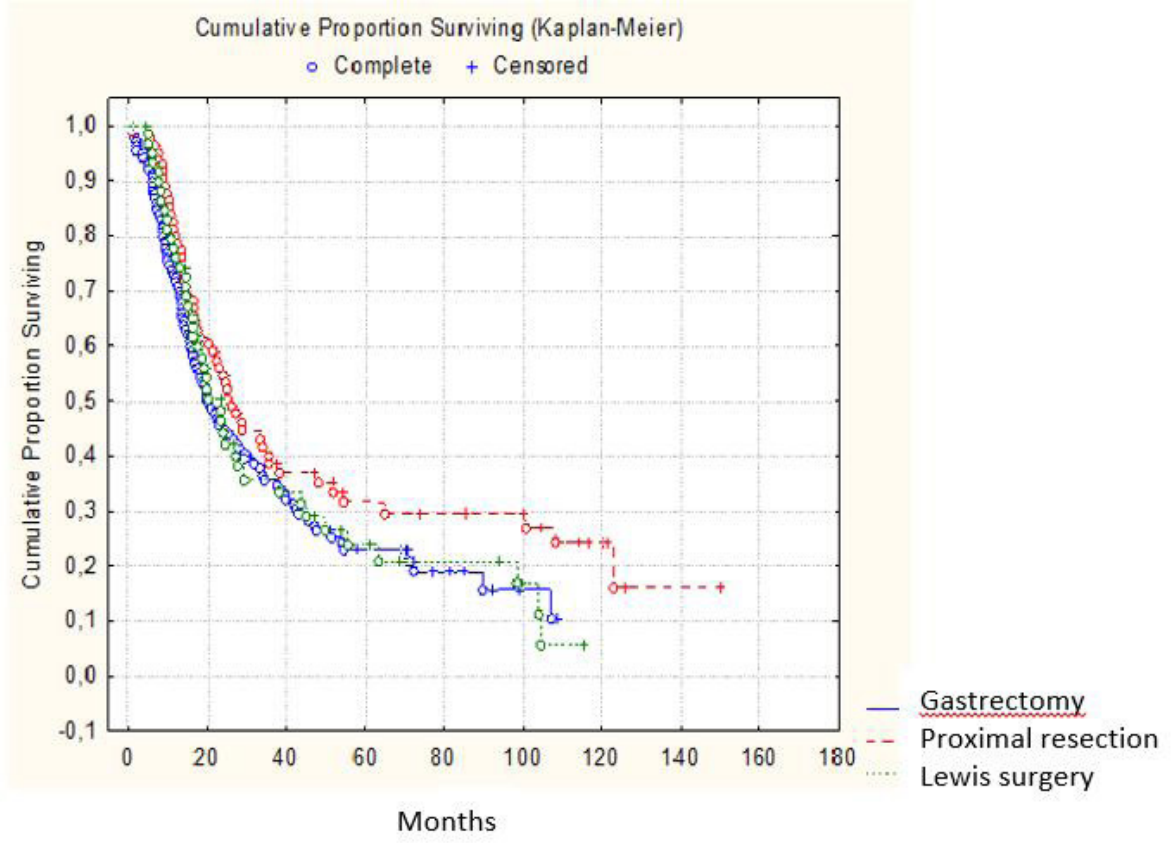

Figure 4: Survival according to the surgery type $(\mathrm{P}>0.05)$

It should be noted that this type of surgery was performed on patients with disease in earlier stages; however, it does not mean that proximal gastric resection should be considered as a "standard" approach.

Analysis of long-term results by the type of gastroesophageal tumor morphological structure confirmed that the difference in survival rates is not statistically significant; however, in cases with undifferentiated or poorly differentiated types of cancer the long-term results of treatment were less satisfactory (Figure 5).

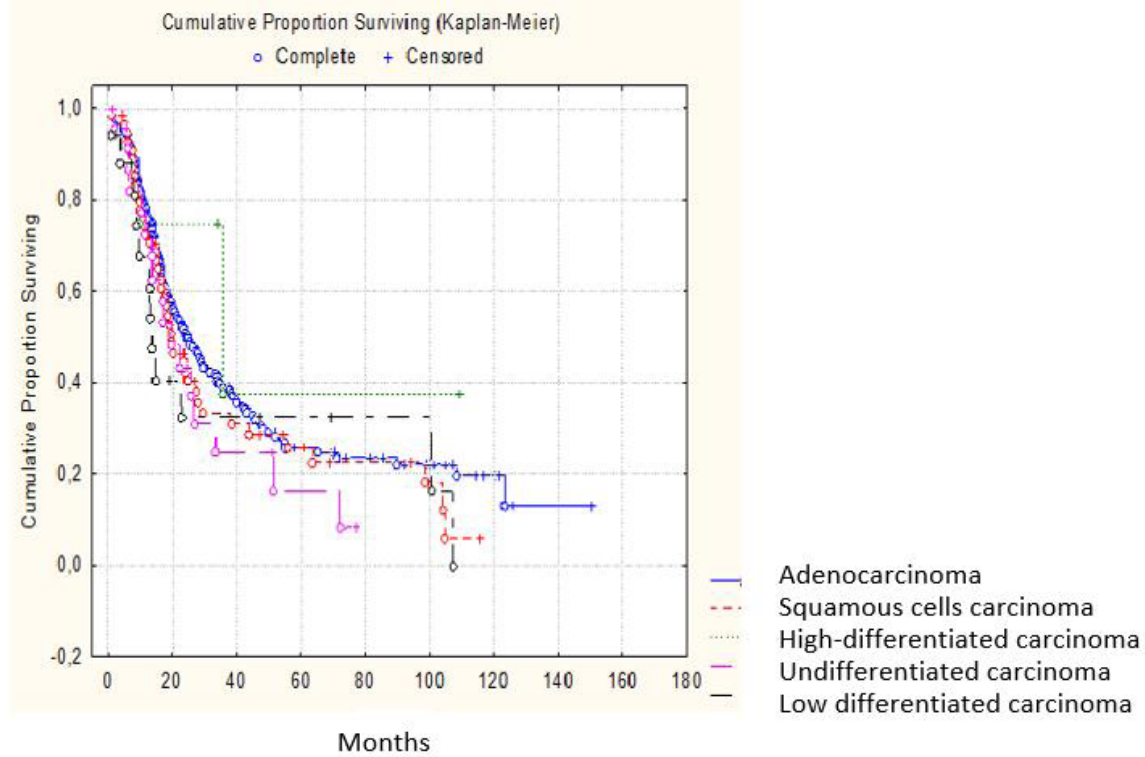

Figure 5: Survival according to the histological tumor type $(\mathrm{P}>0.05)$

Even though the postoperative mortality is relatively low (5.2\%), the long-term results of surgical treatment of gastroesophageal cancer are not satisfactory. Thus, even in the group of patients with favorable major prognostic factors (localized tumor, absence of metastases) the 5- year survival rate did not reach 50\%. By the overall survival, the majority of the performed surgeries should be classified as palliative.

\section{Discussion}

Admittedly, the prospect of improving the long-term outcomes of treatment of gastroesophageal cancer is associated with the development of combined-therapy approaches. While the surgical intervention remains a major treatment modality as a regional therapy, systemic therapy should also be added to the treatment scheme in order to hinder the process of distant metastasis, which is the leading cause of death of patients in the longer time periods. It would be not feasible to improve the long-term results by using 
just a combination of two local interventions (surgery and radiation) due to the high likelihood of the distant metastatic development in 1.5-2 year term after the surgery. These concerns require considering more efficient combined options, such as applying the regional (surgery, radiation) and systemic (chemotherapy) treatments. One of the approaches proposed is perioperative chemotherapy. The UK MRC MAGIC randomized study conducted for cases of gastric cancer in stages II and III revealed that perioperative chemotherapy performed by ECF scheme (epirubicin, cisplatin and continuous intravenous infusion of 5-fluorouracil) improved the 5-year survival rate from $23.0 \%$ to $36.3 \%$ [14]. Similar outcomes were reported in trials conducted by FNCLCC (Féderation Nationale des Centres de Lutte Contre le Cancer) and FFCD (Fédération Francophone de la Cancérologie Digestive) [15]. Based on the results of these studies, perioperative chemotherapy with various schemes of drugs has been extensively adopted as a standard for providing the medical care for gastroesophageal cancer patients in many countries in Europe [16].

The recent ESMO recommendations include adjuvant chemoradiotherapy for stage $\geq$ IB gastroesophageal cancer. A randomized study by the North American Intergroup-0116 demonstrated that five cycles of postoperative chemotherapy with 5-fluorouracil/ leucovorin in combination with radiation therapy ( 45 Gy in 25 fractions within 5 weeks) resulted in improvement of the 5-year overall survival rate by $15 \%$ compared to that of the surgery alone [17]. Even though this treatment approach is considered as standard care in the United States, it is not generally accepted in Europe due to the high toxicity of the therapy. A retrospective study conducted in Holland has demonstrated that chemoradiotherapy reduced local recurrence following the D1 lymph node resection; however it had no statistical significance in improving the outcome following D2 resection [18]. Nonetheless, some other randomized and non- randomized studies indicate potentially positive effect even after optimal D2 lymph node dissection [19-21]. The Asian study (ACTS-GC) of 1059 patients in stages II/III cancer, who undergone D2-D3 lymph node dissection and oral fluoropyrimidine S-1 chemotherapy, demonstrated the increase of the 3-year survival rate from $71.1 \%$ to $80.1 \%$ [22].

Unfortunately, we are lacking sufficient empirical experience in applying the full -scaled neoadjuvant chemoradiotherapy to treating cancer of this localization. In this study we have not conducted the separate assessment of the impact of the chemotherapy on the results of the surgical treatment. In case of disease progression or relapse, the radiotherapy and chemotherapy schemes were prescribed by the multidisciplinary tumor board depending mostly on the patient's condition considering the possible side effects due to high toxicity of drugs.

An evolving and promising approach to cancer treatment is personalized care with customized drug combinations. According to the scientific data, $10-15 \%$ of gastric cancer patients are HER2/neu positive, which implies conducting anti-EGFR targeted therapy. A series of randomized studies demonstrated that including bevacizumab, cetuximab, panitumumab and other antiEGFR drugs in the first-line chemotherapy results in the significant increase in overall survival rates [23-26].

From a theoretical point of view, conducting preoperative adjuvant chemoradiotherapy on patients suited for curative surgery involves certain benefits such as precisely targeted area of radiation and increased intensity of the therapy; however this approach still remains at the clinical research level and its effectiveness is not yet reliably confirmed by randomized studies.

\section{Conclusions}

1. Survival of gastroesophageal cancer patients, who undergone curable surgical treatment is low; after transpleural gastric and esophagus resection the 3 -year survival rate is $37.1 \%$, and the 5 -year survival rate is $26.2 \%$.

2. Prognosis and survival mainly depend on the extent of the tumor spread. For all types of surgeries performed, the survival rate of patients without regional lymph nodes metastases was twice as high as that of patients with lymph nodes metastases. Long- term survival does not depend on the number of affected lymph nodes.

3. Combined and palliative surgeries performed in cases with locally distributed gastroesophageal cancer resulted in the 5- year survival rate of only $10-15 \%$. Nevertheless, for patients with advanced cancer such an intervention is still justified with regards to improved quality of life and prevention of complications such as dysphagia, exhaustion, and hemorrhaging of tumors.

4. Considering the distinctive characteristics of gastroesophageal cancer and its metastatic extension into abdominal and mediastinal lymph nodes, it is advisable to develop separate TNM classification for cancer of this localization.

\section{References}

1. Ferlay J, Steliarova-Foucher E, Lortet-Tieulent J, Rosso S, Coebergh JW, et al. (2013) Cancer incidence and mortality patterns in Europe: estimates for 40 countries in 2012. Eur J Cancer 49: 1374-403.

2. Davydov MI (2007) New surgical techniques in oncology. Annals of the Russian academy of medical sciences 10: 4-9.

3. Chang-Ming H, Bi-Juan L, Hui-Shan L, Xiang-Fu Z, Ping L, et al. (2008) Prognostic impact of metastatic lymph node ratio in advanced gastric cancer from cardia and fundus. World J Gastroenterol 14: 4383-8.

4. Okeanov AE, Moiseyev PI, Levin LF (2014) Statistics of cancer diseases in the Republic of Belarus 2004-2013. Edited by Sukonko OG. Minsk: N.N. Alexandrov National Cancer Centre of Belarus 69-75.

5. Siewert JR, Feith M, Stein HJ (2005) Biologic and clinical variations of adenocarcinoma at the esophago-gastric junction: relevance of a topographic-anatomic subclassification. J Surg Oncol 90: 139-46. 
6. Davydov MI, Turkin IN, Stilidi IS, Polotsky BE, Ter-Ovanesov MD (2003) Cardioesophageal cancer: classification, surgical approach, the main prognostic factors. J N.N. Blokhin Russian Cancer Research Center RAMS 1: 82-8.

7. Chissov VI, Vashakmadze LA, Sidorov DV, Khomyakov VM, Lozhkyn MV (2003) Cancer of the proximal stomach: modern approaches to diagnosis and treatment. J N.N. Blokhin Russian Cancer Research Center RAMS 1: 91-5.

8. DeMeester SR (2006) Adenocarcinoma of the Esophagus and Cardia: A Review of the Disease and Its Treatment. Ann Surg Oncol 13: 12-30.

9. Siewert RJ, Feith M, Werner M, Stein HJ (2000) Adenocarcinoma of the esophagogastric junction: results of surgical therapy based on anatomical/topographic classification in 1,002 consecutive patients. Ann Surg 232: 353-61.

10. Songun I, Putter H, Kranenbarg EM, Sasako M, van de Velde CJ (2010) Surgical treatment of gastric cancer: 15- year follow-up results of the randomised nationwide Dutch D1D2 trial. Lancet Oncol 11: 439-49.

11. Jiang L, Yang KH, Guan QL, Zhao P, Chen Y, et al. (2013) Survival and recurrence free benefits with different lymphadenectomy for resectable gastric cancer: a meta-analysis. J Surg Oncol 107: 807-14.

12. Dikken JL, van Sandick JW, Allum WH, Johansson J, Jensen LS, et al. (2013) Differences in outcomes of oesophageal and gastric cancer surgery across Europe. Br J Surg 100: 83-94.

13. Hulscher JB, van Sandick JW, de Boer AG, Wijnhoven BP, Tijssen JG, et al. (2002) Extended transthoracic resection compared with limited transhiatal resection for adenocarcinoma of the esophagus. N Engl J Med 347: 1662-9.

14. Cunningham D, Allum WH, Stenning SP, Thompson JN, Van de Velde CJ, et al. (2006) Perioperative chemotherapy versus surgery alone for resectable gastroesophageal cancer. N Engl J Med 355: 11-20.

15. Ychou M, Boige V, Pignon JP, Conroy T, Bouché O, et al. (2011) Perioperative chemotherapy compared with surgery alone for resectable gastroesophageal adenocarcinoma: an FNCLCC and FFCD multicenter phase III trial. J Clin Oncol 29: 1715-21.

16. Cunningham D, Starling N, Rao S, Iveson T, Nicolson M, et al. (2008) Capecitabine and oxaliplatin for advanced esophagogastric cancer. N Engl J Med 358: 36-46.

17. Smalley SR, Benedetti JK, Haller DG, Hundahl SA, Estes NC, et al. (2012) Updated analysis of SWOG- directed intergroup study 0116: a phase III trial of adjuvant radiochemotherapy versus observation after curative gastric cancer resection. J Clin Oncol 30: 2327-33.

18. Dikken JL, Jansen EP, Cats A, Bakker B, Hartgrink HH, et al. (2010) Impact of the extent of surgery and postoperative chemoradiotherapy on recurrence patterns in gastric cancer. J Clin Oncol 28: 2430-6.

19. Kim S, Lim DH, Lee J, Kang WK, MacDonald JS, et al. (2005) An observational study suggesting clinical benefit for adjuvant postoperative chemoradiation in a population of over 500 cases after gastric resection with D2 nodal dissection for adenocarcinoma of the stomach. Int J Radiat Oncol Biol Phys 63: $1279-85$.

20. Lee J, Lim do H, Kim S, Park SH, Park JO, et al. (2012) Phase III trial comparing capecitabine plus cisplatin versus capecitabine plus cisplatin with concurrent capecitabine radiotherapy in completely resected gastric cancer with D2 lymph node dissection: the ARTIST trial. J Clin Oncol 30: 268-73.

21. Zhu WG, Xua DF, Pu J, Zong CD, Li T, et al. (2012) A randomized, controlled, multicenter study comparing intensity-modulated radiotherapy plus concurrent chemotherapy with chemotherapy alone in gastric cancer patients with D2 resection. Radiother Oncol 104: 361-6.

22. Sasako M, Sakuramoto S, Katai H, Kinoshita T, Furukawa H, et al. (2011) Five-year outcomes of a randomized phase III trial comparing adjuvant chemotherapy with S-1 versus surgery alone in stage II or III gastric cancer. J Clin Oncol 29: 4387-93.

23. Ohtsu A, Shah MA, Van Cutsem E, Rha SY, Sawaki A, et al. (2011) Bevacizumab in combination with chemotherapy as first-line therapy in advanced gastric cancer: a randomized, double-blind, placebo-controlled phase III study. J Clin Oncol 29: 3968-76.

24. Fuchs CS, Tomasek J, Cho JY, Tomasello G, Goswami C, et al. (2013) REGARD: a phase III, randomized, doubleblinded trial of ramucirumab and best supportive care (BSC) versus placebo and BSC in the treatment of metastatic gastric or gastroesophageal junction (GEJ) adenocarcinoma following disease progression onfirst-line platinum- and/or fluoropyrimidine-containing combination therapy. J Clin Oncol 32: 5s.

25. Lordick F, Kang YK, Chung HC, Salman P, Oh SC, et al. (2013) Capecitabine and cisplatin with or without cetuximab for patients with previously untreated advanced gastric cancer (EXPAND): a randomised, open-label phase 3 trial. Lancet Oncol: 14: 490-9.

26. Waddell T, Chau I, Cunningham D, Gonzalez D, Okines AF, et al. (2013) Epirubicin, oxaliplatin, and capecitabine with or without panitumumab for patients with previously untreated advanced oesophagogastric cancer (REAL3): a randomised, open-label phase 3 trial. Lancet Oncol 14: 481-9.

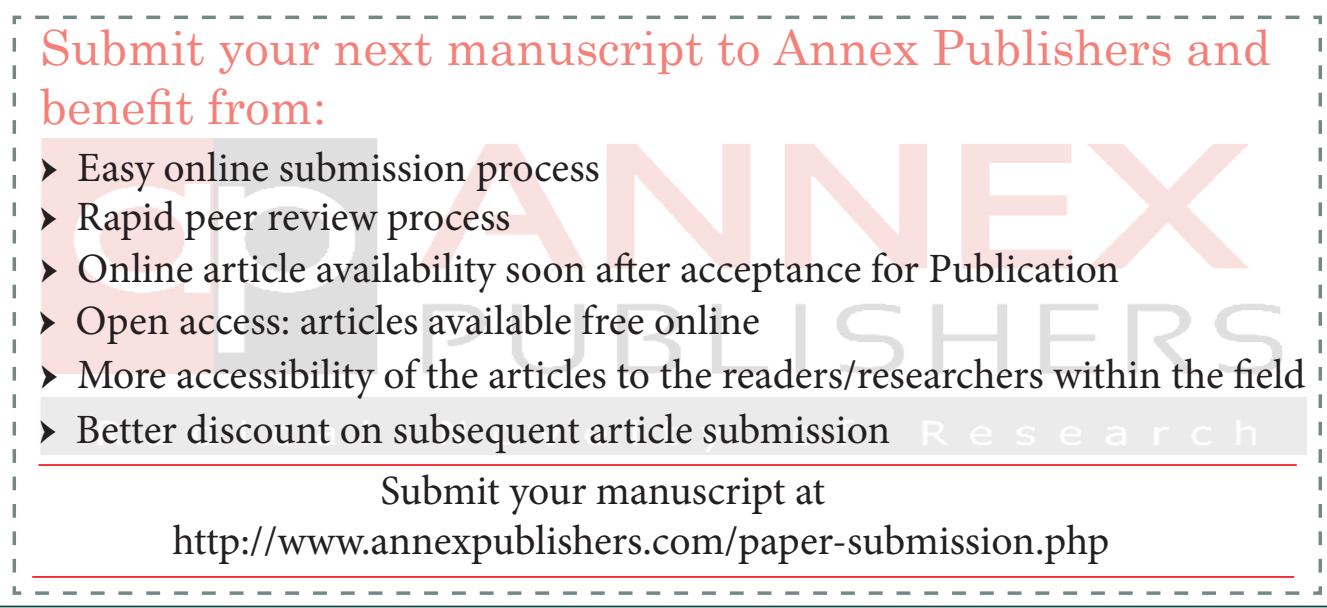

